\title{
Introduction to the Special Issue: COVID-19 and Its Impact on Racial/Ethnic Groups
}

\author{
Gregory N. Price ${ }^{1}$
}

Accepted: 16 November 2020 / Published online: 23 November 2020

(C) Springer Nature Switzerland AG 2020

As of December 2020, the global human Coronavirus (COVID-19) pandemic continued after the first human infections were reported in China in December of 2019. Almost immediately, social scientists aimed their disciplinary gaze on COVID-19 and have attempted to explain the causes and consequences of the COVID-19 pandemic. Early evidence suggested that the incidence of COVID-19 infections among individuals was not neutral with respect to geography, gender, age, and race/ethnicity (Blundell et al. 2020; Cheng et al. 2020; Islam et al. 2020; Kemp et al. 2020; Obeng-Odoom 2020; Rose-Redwood et al. 2020). This special issue of the Journal of Economics, Race, and Policy features contributions from social scientists that provide further insights into how the COVID-19 pandemic intersects with race, ethnicity, gender, religion, and geography. The four contributions, all subject to external critical peer review, while advancing our knowledge on the consequences of COVID-19, also report results that could potentially inform policies to mitigate the disproportionate race, ethnicity, gender, religion, and geography effects of COVID-19.

Ajide, Alimi, Ibrahim, and Nwokolo explore the role of ethno-religious factors and its intersection with political economy in Nigeria. They find that ethnicity and religion intersect in Nigeria so as to condition the incidence of COVID-19. They find for example that the incidence of COVID-19 is relatively higher among the Hausa ethnic group, which appears to be conditioned on the interaction between educational attainment and particular religious beliefs regarding who has the sovereignty of the human soul. Their findings suggest that to be effective, COVID-19 mitigation policies in Nigeria should be conditioned on ethnic/religious considerations.

Gregory N. Price

gnprice@uno.edu

1 Department of Economics and Finance, University of New Orleans, New Orleans, LA 70148, USA
Benitez, Courtmanche, and Yelowitz examine racial and ethnic disparities in confirmed COVID-19 cases across six diverse US cities at the zip code level. Controlling for nonracial/ethnic characteristics, they find evidence that the proportions of Black and Hispanic residents in a ZIP code were associated with higher COVID-19 cases per capita, and the association was even stronger for Hispanic residents. Their findings suggest that the higher death toll of COVID-19 in predominantly Black and Hispanic communities mostly reflects higher case rates, rather than higher fatality rates for confirmed cases. This suggests COVID-19 mitigation policies in the USA should be tailored to recognize the racially/ ethnically disparate rates of COVID-19 infections, relative to associated COVID-19 fatalities.

Gezici and Ozay consider the racialized and gendered effects of the COVID-19 pandemic on the probability of being unemployed in the USA. Their findings cast the unemployment effects of the COVID-19 pandemic in discrimination theories based on unexplained gaps after accounting for observable characteristics of women, men, and different races/ ethnicities and their labor market position. Given the presumption that being employed in a job that is "teleworkable" provides some buffer against being unemployed by the COVID-19 pandemic, a key finding is that Black women and Hispanic women still experience a significantly higher probability of losing their jobs compared to White men even if they are employed in industries with highly teleworkable jobs.

Vargas and Sanchez consider the impact COVID-19 had on the well-being of Latino families with respect to employment, self-employment savings, finances, and psychological coping. With descriptive and inferential methods, the report shows evidence that in the USA, Latinos experienced high job loss, business closures, economic stress, and difficulty making housing payments, and postponed educational and healthrelated services to make ends meet. Their findings suggest that to be effective, COVID-19 mitigation policies in the USA 
should be tailored to recognize the disparate impact the pandemic had on Latino families.

I commend all the authors for making their contributions available to the Journal of Economics, Race, and Policy. Hopefully, the contributions featured in this special issue will be viewed as consequential additions to what we know/will know about the causes/consequences of the COVID-19 pandemic, particularly the extent to which it has effects conditioned on race and ethnicity. Last but not least, it is hoped that the contributions can inform COVID-19 mitigation policies that break any nexus that exists between COVID-19 infections and disparate outcomes conditioned on race/ethnicity.

A special thanks is extended to all the contributors of articles to this special issue, and to the external referees of each contribution. I also thank Gary A. Hoover, the editor of the Journal of Economics, Race, and Policy, for enabling the special issue.

\section{References}

Blundell R, Dias MC, Joyce R, Xiaowei X. COVID-19 and inequalities. Fisc Stud. 2020;41:291-319.

Cheng KJG, Sun Y, Monnat SM. COVID?19 death rates are higher in rural counties with larger shares of blacks and Hispanics. J Rural Health. 2020;36:602-8.

Islam N, Khunti K, Dambha-Miller H, Kawachi I, Marmot M. COVID19 mortality: a complex interplay of sex, gender and ethnicity. Eur J Pub Health. 2020;30:847-8.

Kemp E, Price GN, Fuller NR, Kemp EF. African Americans and COVID-19: beliefs, behaviors and vulnerability to infection. Int J Healthc Manag. 2020;13:303-11.

Obeng-Odoom F. COVID-19, inequality, and social stratification in Africa. Afr Rev Econ Finance. 2020;12:3-37.

Rose-Redwood R, Kitchin R, Apostolopoulou E, Rickards L, Blackman T, Crampton J, et al. Geographies of the COVID-19 pandemic. Dialogues Hum Geogr. 2020;10:97-106.

Publisher's Note Springer Nature remains neutral with regard to jurisdictional claims in published maps and institutional affiliations. 